\title{
High Order Iterative Methods for Matrix Inversion and Regularized Solution of Fredholm Integral Equation of First Kind with Noisy Data
}

\author{
Suzan Cival Buranay ${ }^{1, *}$ and Ovgu Cidar Iyikal ${ }^{1}$ \\ ${ }^{1}$ Department of Mathematics, Faculty of Arts and Sciences, Eastern Mediterranean University, \\ Famagusta, North Cyprus, via Mersin 10, Turkey
}

\begin{abstract}
The motivation of the present work is to propose high order iterative methods with a recurrence formula for approximate matrix inversion and provide regularized solution of Fredholm integral equation of first kind with noisy data by an algorithm using the proposed methods. From the given family of methods of orders $p=7,11,15,19$ are applied to solve problems of Fredholm integral equation of first kind. From the literature, iterative methods of same orders are used to solve the considered problems and numerical comparisons are shown through tables and figures.
\end{abstract}

\section{Introduction}

Consider the system of linear equations

$$
A x=b, A \in R^{N_{1} \times N_{1}}
$$

when, A is nonsingular dense stored matrix. Such a system is commonly solved by Gaussian elimination with pivoting and the accuracy of the computed solution depends primarily on the condition number of the matrix $A$ [1]. The discretization of the ill-posed problems such as the Fredholm integral equations of first kind with smooth kernel results algebraic linear systems possessing a severely ill-conditioned or singular coefficent matrix and these problems are known as linear discrete ill-posed problems. In these problems the solution is very sensitive to perturbations in the data such as measurement or approximation errors. In order to determine a meaningful approximation of the solution of (1) when the coefficient matrix $A$ is severely ill-conditioned one typically replaces the linear system (1) by a nearby system

$$
\widetilde{A} y=\widetilde{b},
$$

that is less sensitive to the perturbations of the right-hand side $b$ and the coefficient matrix $A$ (see [2]-[8]) . Another approach to find a smooth solution to the ill-conditioned system

(1) when $A$ is nonsingular is to use a preconditioner matrix $V$ that approximates $A^{-1}$. The $p$-th order hyperpower methods $p \geq 2$ are a family of well known methods used to approximate the inverse of a nonsingular matrix [9]. Recent studies on iterative methods for computing approximate inverse are but not limited to [10]-[12]. The factorization of hyperpower method of order 7 for computing generalized inverse $A_{T, S}^{(2)}$

$$
V_{m+1}=V_{m}\left(I+\left(T_{m}+T_{m}^{4}\right)\left(I+T_{m}+T_{m}^{2}\right)\right), m=0,1, \ldots .
$$

\footnotetext{
*Corresponding author: suzan.buranay@emu.edu.tr
} 
is proposed in [11]. In [12] several systematic algorithms for the factorizatios of the hyperpower methods of orders 11,15 , and 19

$$
\begin{aligned}
& V_{m+1}=V_{m}\left(I+T_{m}\left(I+\left(T_{m}+T_{m}{ }^{2}+T_{m}^{3}\right)\left(I+T_{m}^{3}+T_{m}^{6}\right)\right)\right), m=0,1, \ldots, \\
& V_{m+1}=V_{m}\left(I+\left(T_{m}+T_{m}{ }^{2}\right)\left(I+\left(T_{m}{ }^{2}+T_{m}^{4}\right)\left(I+T_{m}{ }^{4}+T_{m}^{8}\right)\right)\right), m=0,1, \ldots, \\
& V_{m+1}=V_{m}\left(I+\left(T_{m}+T_{m}{ }^{2}\right)\left(I+T_{m}{ }^{2}+T_{m}{ }^{4}\right)\left(I+T_{m}{ }^{6}+T_{m}{ }^{12}\right)\right), m=0,1, \ldots,
\end{aligned}
$$

are given, respectively to approximate the outer inverse.

In this study for a given integer $k \geq 1$, we propose high order iterative methods of orders $p=4 k+3$, using a recurrence formula to compute approximate matrix inverse and use the methods of orders $p=7,11,15,19$ from this family to obtain regularized solution of Fredholm Integral Equations of first kind such as the harmonic continuation problem.

\section{A Family of Methods with Recurrence Formula}

Let $I$ denote the $N_{1} \times N_{1}$ unit matrix and $A \in R^{N_{1} \times N_{1}}$ be nonsingular matrix. We denote the approximate inverse of $A$ at the $m$-th iteration by $V_{m}$, and the residual matrix by $T_{m}=I-A V_{m}$. Let

$$
\begin{aligned}
& \aleph\left(T_{m}\right)=T_{m} T_{m}, \Omega\left(T_{m}\right)=T_{m}+\aleph\left(T_{m}\right), \\
& \Gamma\left(T_{m}\right)=\aleph\left(T_{m}\right) \aleph\left(T_{m}\right), \Psi\left(T_{m}\right)=\aleph\left(T_{m}\right)+\Gamma\left(T_{m}\right) .
\end{aligned}
$$

We call Family Generator Function to the matrix valued function $\Phi_{p}\left(T_{m}\right)$, defined as

$$
\Phi_{p}\left(T_{m}\right)=\Omega\left(T_{m}\right)\left(I+\Psi\left(T_{m}\right)\left[I+\Gamma\left(T_{m}\right)\left(I+\Gamma\left(T_{m}\right)\left(I+\Gamma\left(T_{m}\right)(\ldots)\right)\right)\right]\right),
$$

with $\left(I+\Gamma\left(T_{m}\right)\right)$ is 1 nested loop, $\left(I+\Gamma\left(T_{m}\right)\right)\left(I+\Gamma\left(T_{m}\right)\right)$ is 2 nested loop, (8) can be given by the following recurrence formula

$$
\begin{aligned}
& P_{0}=I, P_{j}=I+\Gamma\left(T_{m}\right) P_{j-1}, j=1, \ldots, k-1, \\
& \hat{\Phi}_{p}\left(T_{m}\right)=\Phi_{p}\left(T_{m}\right)=\Omega\left(T_{m}\right)\left(I+\Psi\left(T_{m}\right) P_{k-1}\right) .
\end{aligned}
$$

For an integer $k \geq 1$ and the corresponding Family Generator Function $\hat{\Phi}_{p}\left(T_{m}\right), p=4 k+1$, we proposed

$$
V_{m+1}=V_{m}\left(I+\hat{\Phi}_{p}\left(T_{m}\right)\right), m=0,1, \ldots
$$

the family of methods for matrix inversion.

Theorem : Let $A \in R^{N_{1} \times N_{1}}$ be a nonsingular matrix. For a given $k \geq 1$ integer if the method (10) is used, the necessary and sufficient condition for the convergence of (10) to $A^{-1}$ is that $\rho\left(T_{0}\right)<1$ holds, where $\rho$ is spectral radius, $T_{0}=I-A V_{0}$ and $V_{0}$ is the initial approximation.

\section{Computational Complexity and Algorithm for Regularized Solution}

When the two factorizations of $p$-th order hyperpower method have same asymptotic convergence factor ACF [10] values then it is necessary to use an other quantity which measures the efficiency of the hyperpower method both with respect to matrix by matrix multiplications (MMs) and matrix by matrix additions (Mas). Let $\kappa$ be the number of MMs and $p$ be the order of the hyperpower method. Let $v$ be the number of Mas and $\gamma$ be the number of matrix additions by identity. We define the Asymptotic Convergence Values by 


$$
A C V=\left(\frac{\kappa}{\ln p}, \frac{v}{\ln p}, \frac{\gamma}{\ln p}\right) .
$$

Table 1 shows the rounded $A C V$ of the proposed methods for the orders $p=7,11,15,19$ and of the methods (3), (4), (5), (6). We denote the methods (10) for $k=1, k=2, k=3, k=4$ by $M_{1}, M_{3}, M_{5}, M_{7}$, respectively and the methods (3), (4), (5), (6) by $M_{2}, M_{4}, M_{6}, M_{8}$, respectively.

\begin{tabular}{l|llll}
\hline Method & $\kappa$ & $v$ & $\gamma$ & $A C V$ \\
\hline $\mathrm{M}_{1}$ & 5 & 2 & 3 & $(2.5695,1.0278,1.5417)$ \\
$\mathrm{M}_{2}$ & 5 & 2 & 3 & $(2.5695,1.0278,1.5417)$ \\
$p=7$ in $[10]$ & 6 & 1 & 4 & $(3.083,0.5139,2.0556)$ \\
$\mathrm{M}_{3}$ & 6 & 2 & 4 & $(2.5022,0.8341,1.6681)$ \\
$\mathrm{M}_{4}$ & 7 & 3 & 4 & $(2.9102,1.2511,1.6681)$ \\
$\mathrm{M}_{5}$ & 7 & 2 & 5 & $(2.5849,0.7385,1.8463)$ \\
$\mathrm{M}_{6}$ & 7 & 3 & 4 & $(2.5849,1.1078,1.4771)$ \\
$\mathrm{M}_{7}$ & 8 & 2 & 6 & $(2.7170,0.6792,2.0377)$ \\
$\mathrm{M}_{8}$ & 8 & 3 & 4 & $(2.7170,1.0189,1.3584)$ \\
$p=19$ in $[10]$ & 10 & 2 & 5 & $(3.3962,0.6792,1.6981)$ \\
\hline
\end{tabular}

Table 1. ACV for the proposed family of methods (10) and for the methods from the literature [10], [11] and [12] of the orders $p=7,11,15,19$.

We consider the perturbed system (2) in the following form

$$
(A+\Delta A) y=b+\Delta b
$$

where $\tilde{A}=A+\Delta A, \tilde{b}=b+\Delta b$ and $\Delta A$ is the perturbation in $A, \Delta b$ is the perturbation in $b$. The applicability of the proposed methods for solving the perturbed system (12) is given by the following algorithm.

Algorithm 1 : When $A \in R^{N_{1} \times N_{1}}$ is nonsingular matrix for a given integer $k \geq 1$ and a predescribed accuracy $\varepsilon>0$, the approximate inverse $V_{\tilde{m}}$ of $\tilde{A}$ in (12) is found by using the methods (10) with $\tilde{m}$ iterations to reach the accuracy $\frac{\left\|r_{\tilde{m}}\right\|_{\infty}}{\|\tilde{b}\|_{\infty}} \leq \varepsilon$ where $r_{\tilde{m}}=\tilde{b}-\tilde{A} y_{\tilde{m}}$. Here $y_{\tilde{m}}=V_{\tilde{m}} b$ is the regularized solutions. This algorithm is given analogous to Algorithm 1 in $[10]$.

\section{Numerical Results}

The numerical analysis of the proposed methods (10) of orders $p=7,11,15,19$ are provided by the application on two examples of Fredholm integral equation of first kind. All the computations are performed using a personal computer with properties Intel(R) Core(TM)-2DUO CPUT5870@2.00GHz and Fortran programs in double precision.

Harmonic Continuation Problem: We consider the harmonic continuation problem [1]:

$$
\frac{1}{2 \pi} \int_{0}^{2 \pi} \frac{1-r^{2}}{1-2 r \cos (\theta-\varphi)+r^{2}} h(\varphi) d \varphi=f(\theta),
$$

for the same data used by Franklin [8] where, for $|z|=r=0.5$, 
$h(\varphi)=\cos 3 \varphi-\cos \varphi+[\sin (\cos \varphi)][\cos (\sin \varphi)], f(\theta)=\frac{1}{8} \cos 3 \theta-\frac{1}{2} \cos \theta+\left[\sin \left(\frac{1}{2} \cos \theta\right)\right]\left[\cos \left(\frac{1}{2} \sin \theta\right)\right]$,

Using the quadrature nodes $\varphi_{j}=\frac{2 \pi j}{N_{1}}, j=1, \ldots, N_{1}$, evaluating $f(\theta)$ at the points $\theta_{i}=\frac{2 \pi i}{N_{1}}, \mathrm{i}=1, \ldots, N_{1}$ and by discretizing (13) we obtain the algebraic linear system (1) as

$$
A_{i, j}=\frac{3}{N_{1}\left(5-4 \cos 2 \pi(i-j) / N_{1}\right)}, b_{j}=f\left(\frac{2 \pi j}{N_{1}}\right) .
$$

Consider a noisy vector $\tilde{b}$ and a noisy matrix $\tilde{A}$ with available noise levels $\delta_{b}$ and $\delta_{A}$, respectively such that

$$
\|\tilde{b}-b\|_{2}=\|\Delta b\|_{2} \leq \delta_{b} \text { and }\|\tilde{A}-A\|_{2}=\|\Delta A\|_{2} \leq \delta_{A} .
$$

We take $N_{1}=50, \delta_{b}=10^{-11}, \delta_{A}=0.5\left(\delta_{b}\right)^{1.5}$ and apply the Algorithm 1 for the methods $M_{i}, i=1, \ldots, 8$ when $V_{0}=\frac{\tilde{A}^{T}}{\|\tilde{A}\|_{1}\|\tilde{A}\|_{\infty}}$ and $\varepsilon=5 \times 10^{-11}$. Table 2 presents the CPU time in seconds, iteration numbers $\tilde{m}$ and relative norms of the errors obtained by Algorithm 1 using the methods $M_{i}, i=1, \ldots, 8$ for the harmonic continuation problem.

\begin{tabular}{l|llll}
\hline Methods & $C P U$ & $\tilde{m}$ & $\left\|x-y_{\tilde{m}}\right\|_{\infty}$ & $\frac{\left\|x-y_{\tilde{m}}\right\|_{2}}{\|x\|_{2}}$ \\
\hline $\mathrm{M}_{1}$ & 0.094 & 8 & $7.5780278 \mathrm{E}-009$ & $7.6071543 \mathrm{E}-009$ \\
$\mathrm{M}_{2}$ & 0.094 & 8 & $7.5778557 \mathrm{E}-009$ & $7.6070067 \mathrm{E}-009$ \\
$\mathrm{M}_{3}$ & 0.094 & 7 & $3.7195719 \mathrm{E}-010$ & $3.2294976 \mathrm{E}-010$ \\
$\mathrm{M}_{4}$ & 0.140 & 7 & $3.7184901 \mathrm{E}-010$ & $3.2282504 \mathrm{E}-010$ \\
$\mathrm{M}_{5}$ & 0.094 & 6 & $2.0519901 \mathrm{E}-009$ & $1.9954705 \mathrm{E}-009$ \\
$\mathrm{M}_{6}$ & 0.095 & 6 & $2.0521896 \mathrm{E}-009$ & $1.9954422 \mathrm{E}-009$ \\
$\mathrm{M}_{7}$ & 0.109 & 6 & $1.0521583 \mathrm{E}-010$ & $9.7355378 \mathrm{E}-011$ \\
$\mathrm{M}_{8}$ & 0.140 & 6 & $1.0616301 \mathrm{E}-010$ & $9.7465166 \mathrm{E}-011$ \\
\hline
\end{tabular}

Table.2. CPU times in seconds, iteration numbers $\tilde{m}$ and relative norms of the errors obtained by Algorithm 1 using the methods $M_{i}, i=1, \ldots, 8$ for the harmonic continuation problem, when $N_{1}=50$ and $\delta_{b}=10^{-11}, \delta_{A}=0.5\left(\delta_{b}\right)^{1.5}$.

Phillip's Problem: We consider the Fredholm integral equation of first kind $\int_{-6}^{6} k(s, t) h(t) d t=f(s)$ discussed in [13], where its solution, kernel and the right-hand side are

$$
\begin{aligned}
& h(t)=\left\{\begin{array}{l}
1+\cos \left(\frac{\pi}{3} t\right), \text { if }|t|<3 \\
0, \quad \text { otherwise }
\end{array}\right. \\
& k(s, t)=h(s-t), f(s)=(6-|s|)\left(1+\frac{1}{2} \cos \left(\frac{\pi}{3} s\right)\right)+\frac{9}{2 \pi} \sin \left(\frac{\pi}{3}|s|\right),
\end{aligned}
$$

respectively. We discretize (17) by taking the quadrature nodes $t_{j}=-6+\frac{12 j}{N_{1}}, j=1, \ldots, N_{1}$ and evaluating $f(s)$ at the points $s_{i}=-6+\frac{12 i}{N_{1}}, i=1, \ldots, N_{1}$ for $N_{1}=64$. We apply Algorithm 1 using the methods $M_{i}, i=1, \ldots, 8$ for $\delta_{b}=10^{-7}, \quad \delta_{A}=0.5\left(\delta_{b}\right)^{1.5}$ when 
$V_{0}=\frac{\tilde{A}^{T}}{\|\tilde{A}\|_{1}\|\tilde{A}\|_{\infty}}, \quad \varepsilon=5 \times 10^{-7}$. The CPU time in seconds, iteration numbers $\tilde{m}$ and relative norms of the errors for the Phillip's problem are given in Table 3.

\begin{tabular}{l|llll}
\hline Methods & $C P U$ & $\tilde{m}$ & $\left\|x-y_{\tilde{m}}\right\|_{\infty}$ & $\frac{\left\|x-y_{\tilde{m}}\right\|_{2}}{\|x\|_{2}}$ \\
\hline $\mathrm{M}_{1}$ & 0.078 & 8 & $5.0460857 \mathrm{E}-004$ & $3.6367029 \mathrm{E}-004$ \\
$\mathrm{M}_{2}$ & 0.078 & 8 & $5.0460858 \mathrm{E}-004$ & $3.6367029 \mathrm{E}-004$ \\
$\mathrm{M}_{3}$ & 0.078 & 7 & $2.8448691 \mathrm{E}-004$ & $1.7447789 \mathrm{E}-004$ \\
$\mathrm{M}_{4}$ & 0.093 & 7 & $2.8448691 \mathrm{E}-004$ & $1.7447789 \mathrm{E}-004$ \\
$\mathrm{M}_{5}$ & 0.077 & 6 & $3.4897422 \mathrm{E}-004$ & $2.4563953 \mathrm{E}-004$ \\
$\mathrm{M}_{6}$ & 0.078 & 6 & $3.4897422 \mathrm{E}-004$ & $2.4563953 \mathrm{E}-004$ \\
$\mathrm{M}_{7}$ & 0.077 & 6 & $1.5296893 \mathrm{E}-004$ & $1.0051624 \mathrm{E}-004$ \\
$M_{8}$ & 0.078 & 6 & $1.5296893 \mathrm{E}-004$ & $1.0051624 \mathrm{E}-004$ \\
\hline
\end{tabular}

Table.3. CPU times in seconds, iteration numbers $\tilde{m}$ and relative norms of the errors obtained by Algorithm 1 using the methods $M_{i}, i=1, \ldots, 8$ for the Phillip's problem when

\section{Conclusion}

$$
N_{1}=64 \text { and } \delta_{b}=10^{-7}, \delta_{A}=0.5\left(\delta_{b}\right)^{1.5} \text {. }
$$

The best error of numerical solution occurred in [1] for the Harmonic continuation problem by singular value decomposition when $A \in R^{50 \times 50}$ was approximately $10^{-3}$ using single precision. The minimum relative error given in [13] for Phillip's problem when $A \in R^{64 \times 64}$ is $1.84 \times 10^{-3}$. Comparing with the results in Table 2 and Table 3 proposed Algorithm 1 gives accurate results. Furthermore, the methods $M_{3}, M_{5}, M_{7}$ require less CPU times then the methods $M_{4}, M_{6}, M_{8}$, respectively.

\section{References}

1. J.M.Varah, SIAM, 10(2), 257-267 (1973).

2. P.C. Hansen, SIAM, 4 (1998).

3. D. Colton, R. Kress, Springer, New York (1992).

4. D.D. Ang, R. Gorenflo, V.K. Le, D.D. Trong, 1792 Springer Berlin (2002).

5. F.S. V. Bazán, J.Sci Comput 63, 163-184 (2015).

6. C.W. Groetsch, Research notes in Mathematics 105, Pitman, Boston (1984).

7. A.N. Tikhonov, Soviet Math. Dokl. 4, 1036-1038 (1963).

8. J.N. Franklin, Journal of Mathematical Analysis and Appl., 31, 682-716 (1970).

9. A. Ben-Israel, T. N. E.Greville, Theory and Applications, Springer, (2003).

10. S.C. Buranay, D. Subasi, O.C. Iyikal, Numer. Linear Algebra Appl., 24 (6), (2017).

11. F. Soleymani, Numerical Algorithms, 69 569-578 (2015).

12. F.Soleymani, P.S.Stanimirović, F.K.Haghani, Linear Algebra \& its Appl. 484, 477-495 (2015).

13. M. Rezghi, S. M. Hosseini, Journal of Computational and Applied Mathematics, 231, 914-924 (2009) . 